\title{
Mathematical Model of Two Phase Flow in Natural Draft Wet-Cooling Tower Including Flue Gas Injection
}

\author{
Tomáš Hyhlík ${ }^{1, a}$ \\ ${ }^{1}$ CTU in Prague, FME, Department of Fluid Dynamics and Thermodynamics, Technická 4, 16607 Prague
}

\begin{abstract}
The previously developed model of natural draft wet-cooling tower flow, heat and mass transfer is extended to be able to take into account the flow of supersaturated moist air. The two phase flow model is based on void fraction of gas phase which is included in the governing equations. Homogeneous equilibrium model, where the two phases are well mixed and have the same velocity, is used. The effect of flue gas injection is included into the developed mathematical model by using source terms in governing equations and by using momentum flux coefficient and kinetic energy flux coefficient. Heat and mass transfer in the fill zone is described by the system of ordinary differential equations, where the mass transfer is represented by measured fill Merkel number and heat transfer is calculated using prescribed Lewis factor.
\end{abstract}

\section{Introduction}

Various models of natural draft cooling tower flow, heat and mass transfer exist. Classical approach is described in reference [1], where simple algebraic draft equation is used to calculate moist air mass flow rate. An example of more complex approach based on commercial CFD code is the work [2]. As an example of other code can be mentioned e.g. work [3].

Current work can be understood as continuation of previous work of author, see e.g. [4], [5]. This work is leading to relatively complex CFD model of natural draft cooling tower flow, heat and mass transfer.

The main benefit of current model is possibility to simulate supersaturated moist air. In the case where ambient moist air is undersaturated or supersaturated is supersaturated moist air typically present in the cooling tower and the plume outside cooling tower exist. The present model provides data to estimate plume.

Another benefit of present model is the inclusion of flue gas injection. Flue gas injection has contradictory effect on natural draft wet-cooling tower mass flow rate and thereby on water cooling. From one point of view is flue gas injection reducing cross sectional area of cooling tower and thus reduces mass flow rate, but from the other point of view it creates ejector effect which increases mass flow rate. Last but not least, the inclusion of momentum and energy flux coefficients into the governing equation generalizes the model and allows to include multidimensional effect in quasi one-dimensional model.

\section{Mathematical model of moist air flow}

Lets assume homogeneous equilibrium model of two phase flow, where the two phases are well mixed and have the same velocity. Furthermore the thermal equilibrium of both phases is assumed. Thermodynamic equilibrium implies in

\footnotetext{
a e-mail: tomas.hyhlik@fs.cvut.cz
}

the case of two phase flow, that mixture of water vapour and dry air is saturated. Water liquid has the temperature of the mixture and the pressure of mixture. The water is sub-cooled because mixture pressure is higher then water vapour partial pressure. This model is the kind of homogeneous equilibrium model [6] of two phase flow applied to case where the gas phase is the mixture of condensable and noncondensable components.

\subsection{Mass fractions}

A key step is the definition mass fractions. Overall density of two phase mixture can be expressed using void fraction of gas phase as [6]

$$
\rho=\alpha \rho_{g}+(1-\alpha) \rho_{l},
$$

where $\alpha$ is volumetric void fraction at specific point $x_{i}$ at time $\tau$, i.e. the volume of gas phase $V_{g}$ over the whole $V_{g}+$ $V_{l}$

$$
\alpha\left(x_{i}, \tau\right)=\frac{\mathrm{d} V_{g}\left(x_{i}, \tau\right)}{\mathrm{d} V_{g}\left(x_{i}, \tau\right)+\mathrm{d} V_{l}\left(x_{i}, \tau\right)},
$$

where $\rho_{l}$ is the density of water liquid and $\rho_{g}$ the density of gas phase which is a sum of dry air density $\rho_{a}$ and the density of water vapour

$$
\rho_{g}=\rho_{a}+\rho_{v} .
$$

Specific humidity of supersaturated moist air is

$$
x_{s}=\frac{\alpha \rho_{v}+(1-\alpha) \rho_{l}}{\alpha \rho_{a}}=\frac{\rho_{v}}{\rho_{a}}+\frac{1-\alpha}{\alpha} \frac{\rho_{l}}{\rho_{a}}
$$

and the part corresponding to the gas phase is

$$
x=\frac{\alpha \rho_{v}}{\alpha \rho_{a}}=\frac{\rho_{v}}{\rho_{a}} .
$$

The case of supersaturated moist air is corresponding with $\alpha<1$ and specific humidity of gas phase is equal to saturated specific humidity $x=x^{\prime \prime}$. The mass fraction of dry 
air is in the case of supersaturated moist air equal to

$$
w_{a}=\frac{\alpha \rho_{a}}{\rho}=\left[1+\frac{\rho_{v}}{\rho_{a}}+\frac{1-\alpha}{\alpha} \frac{\rho_{l}}{\rho_{a}}\right]^{-1}=\frac{1}{1+x_{s}},
$$

water vapour mass fraction is defined as

$$
w_{v}=\frac{\alpha \rho_{v}}{\rho}=\left[1+\frac{\rho_{a}}{\rho_{v}}+\frac{1-\alpha}{\alpha} \frac{\rho_{l}}{\rho_{v}}\right]^{-1}=\frac{x}{1+x_{s}}
$$

and water liquid mass fraction is

$$
w_{l}=\frac{(1-\alpha) \rho_{l}}{\rho}=\left[1+\frac{\alpha}{1-\alpha} \frac{\rho_{a}+\rho_{v}}{\rho_{l}}\right]^{-1} .
$$

It is possible to express water liquid mass fraction also as

$$
w_{l}=\frac{x_{s}-x}{1+x_{s}} .
$$

Due to the utilization of void fraction in the definitions of individual mass fractions it is possible to show that

$$
w_{a}+w_{v}+w_{l}=1 \text {. }
$$

The ratio of gas mass fraction to liquid mass fraction can be expressed as

$$
\frac{\alpha \rho_{g}}{(1-\alpha) \rho_{l}}=\frac{\alpha\left(\rho_{a}+\rho_{v}\right)}{(1-\alpha) \rho_{l}}=\frac{w_{a}+w_{v}}{1-w_{a}-w_{v}}
$$

and

$$
\frac{\alpha \rho_{g}}{(1-\alpha) \rho_{l}}=\frac{1+x}{x_{s}-x},
$$

where specific humidity is

$$
x=\frac{w_{v}}{w_{a}} .
$$

\subsection{Governing equations}

Mass balance equations of dry air, water vapour and water liquid are in this section written without diffusion term, which is mostly negligible. The overall mass of dry air in the two phase mixture can be expressed as

$$
m_{a}=\int_{V}\left[\alpha \rho_{a}+(1-\alpha) \rho_{l} w_{a, l}\right] \mathrm{d} V=\int_{V} \varphi_{a} \mathrm{~d} V,
$$

where $w_{a, l}$ is mass fraction of dry air in the water liquid and $\varphi_{a}$ can be expressed also as

$$
\varphi_{a}=\alpha \rho_{g} w_{a, g}+(1-\alpha) \rho_{l} w_{a, l},
$$

where $w_{a, g}$ is mass fraction of dry air in the mixture of dry air and saturated water vapour

$$
w_{a, g}=\frac{w_{a}}{w_{a}+w_{v}} .
$$

Dry air mass balance can by written as

$$
\frac{\partial \varphi_{a}}{\partial \tau}+\frac{\partial\left(\varphi_{a} v_{i}\right)}{\partial x_{i}}=0
$$

where $v_{i}$ is velocity vector. The solubility of dry air in the water liquid can be neglected (i.e. $w_{a, l}=0$ ) and dry air mass balance can be simplified to

$$
\frac{\partial\left(\alpha \rho_{a}\right)}{\partial \tau}+\frac{\partial\left(\alpha \rho_{a} v_{i}\right)}{\partial x_{i}}=0,
$$

or

$$
\frac{\partial\left(\alpha \rho_{g} w_{a, g}\right)}{\partial \tau}+\frac{\partial\left(\alpha \rho_{g} w_{a, g} v_{i}\right)}{\partial x_{i}}=0 .
$$

Water vapour mass balance can be expressed as

$$
\frac{\partial\left(\alpha \rho_{v}\right)}{\partial \tau}+\frac{\partial\left(\alpha \rho_{v} v_{i}\right)}{\partial x_{i}}=\sigma_{v}\left(x_{i}\right)
$$

where $\sigma_{v}$ is density of water vapour mass source. Liquid phase mass balance is

$$
\frac{\partial\left[(1-\alpha) \rho_{l}\right]}{\partial \tau}+\frac{\partial\left[(1-\alpha) \rho_{l} v_{i}\right]}{\partial x_{i}}=\sigma_{l}\left(x_{i}\right),
$$

where $\sigma_{l}$ is density of water liquid mass source and

$$
\sigma_{v}\left(x_{i}\right)=-\sigma_{l}\left(x_{i}\right)
$$

in the case of moist air flow without external sources of both water vapour and water liquid. Overall mass balance equation of supersaturated moist air can be obtained by adding of equations (18), (20) and (21)

$$
\frac{\partial \rho}{\partial \tau}+\frac{\partial\left(\rho v_{i}\right)}{\partial x_{i}}=0
$$

External sources of water vapour or water liquid are not included in the previous equation. Overall momentum balance can be written as

$$
\frac{\partial\left(\rho v_{i}\right)}{\partial \tau}+\frac{\partial}{\partial x_{k}}\left[\rho v_{i} v_{k}-\sigma_{i k}\right]=-\rho g \delta_{i 3}-\zeta \rho \frac{|v|^{2}}{2} \delta_{i 3},
$$

where $\sigma_{i k}$ is stress tensor, the first term on the right represents volume force connected with gravity in the direction $-z$ an the second term allows to add minor losses in the $z$ direction. Overall energy equation is

$$
\frac{\partial(\rho e)}{\partial \tau}+\frac{\partial}{\partial x_{k}}\left[\rho v_{k} e-\sigma_{k j} v_{j}+\dot{q}_{k}\right]=-g \rho v_{3},
$$

where total energy is the sum of internal energy and kinetic energy $e=u+\frac{v_{k} v_{k}}{2}$ and $\dot{q}_{k}$ is diffusion coefficient driven heat flux. The term on the right hand side is density of source term which is connected with gravity force.

We have the system of equations (18), (20), (21), (24) and (25) describing the flow of supersaturated moist air. The evaluation of source term $\sigma_{v}$ appears to be one of the complication of the model, but it is possible to replace one of mass balance equations using overall mass balance equation (23) and evaluation of water vapour mass fraction should be based on thermodynamic relations for saturated moist air. Finally it is possible to formulate the system of governing equations where there are only overall continuity equation (23), overall momentum balance (24), dry air continuity equation (18) and overall equation of energy (25). The system of governing equations has to be closed using the definition of internal energy and using the equation of state. 


\subsection{Thermodynamic parameters}

Internal energy $u$ is defined as

$$
u=\frac{1}{\rho}\left[\alpha \rho_{g} u_{g}+(1-\alpha) \rho_{l} u_{l}\right]
$$

where $u_{l}$ is internal energy of water liquid and internal energy of the mixture of dry air and water vapour is

$$
u_{g}=\frac{1}{\rho_{g}}\left(\rho_{a} u_{a}+\rho_{v} u_{v}\right)=u_{a} w_{a, g}+\left(1-w_{a, g}\right) u_{v},
$$

where $u_{a}$ is internal energy of dry air, $u_{v}$ is internal energy of water vapour and $w_{a, g}(p, t)$ is mass fraction of dry air in the mixture of dry air and saturated water vapour. Finally can be internal energy $u$ expressed as

$$
u=c_{v_{s}}(p, t) t+w_{v}(p, t)\left(l_{T R}-\frac{p_{T R}}{\rho_{T R}}\right),
$$

where $l_{T R}$ is heat of vaporisation at the triple point, $p_{T R}$ is the triple point pressure and $\rho_{T R}$ is the density at the triple point. Temperature in degrees of Celsius is $t$. Mixture specific heat capacity is

$$
c_{v_{s}}(p, t)=w_{a} c_{v_{a}}+w_{v}(p, t) c_{v_{v}}+\left[1-w_{a}-w_{v}(p, t)\right] c_{v_{w}},
$$

where $c_{v_{a}}, c_{v_{v}}$ and $c_{v_{w}}$ are constant volume specific heat capacities of dry air, water vapour and water liquid respectively. Equation of state can be written as

$$
p=r_{s}(p, t) \rho_{g}(p, t) T(p, t),
$$

where $r_{s}$ is gas constant of the mixture of dry air and water vapour

$$
r_{s}(p, t)=\frac{w_{a}}{w_{a}+w_{v}(p, t)} r_{a}+\frac{w_{v}(p, t)}{w_{a}+w_{v}(p, t)} r_{v}
$$

and temperature in Kelvins can be expressed as

$$
T(p, t)=\frac{u-w_{v}(p, t)\left(l_{T R}-\frac{p_{T R}}{\rho_{T R}}\right)}{c_{v_{s}}(p, t)}+273.15 .
$$

The key part of this model of two phase flow is resolving the temperature and pressure based on the equations (28) and (30). The two equations for pressure and temperature are non-linear mainly because water vapour mass fraction is the function of saturation pressure at given temperature

$$
w_{v}(p, t)=0.622 w_{a} \frac{p_{v}(t)}{p-p_{v}(t)} .
$$

The saturation pressure at given temperature can be calculated by using IAPWS formulation [7].

\subsection{Quasi one-dimensional model of natural draft wet-cooling tower including flue gas injection}

The system of governing equations can be written for the case of quasi one-dimensional flow as

$$
\frac{\partial(\mathbf{W} A)}{\partial \tau}+\frac{\partial(\mathbf{F} A)}{\partial z}=\mathbf{Q}_{n d}+\mathbf{Q}_{f g}
$$

where $A(z)$ is cross sectional area of the cooling tower, $z$ is spatial coordinate and $\tau$ is temporal coordinate. Lets assume the moist air flow in the direction of $z$ axis. The first equation is overall continuity equation, the second is overall momentum equation, the third is mass balance of dry air and the last equation is balance of energy. Vector of conservative variables $\mathbf{W}$ and vector of fluxes $\mathbf{F}$ are

$$
\mathbf{W}=\left[\begin{array}{c}
\rho \\
\rho v \\
\rho w_{a} \\
\rho e
\end{array}\right], \mathbf{F}=\left[\begin{array}{c}
\rho v \\
\rho v^{2} \beta+p \\
\rho w_{a} v \\
\left\{\rho\left(u+\gamma \frac{v^{2}}{2}\right)+p\right\} v
\end{array}\right],
$$

where $\beta$ is momentum flux coefficient and $\gamma$ is kinetic energy flux coefficient taking into account non-uniformity of velocity profile and $\gamma$ is kinetic energy flux coefficient

$$
\beta=\frac{A \int_{A} v^{2} \mathrm{~d} A}{\left(\int_{A} v \mathrm{~d} A\right)^{2}}, \gamma=\frac{A^{2} \int_{A} v^{3} \mathrm{~d} A}{\left(\int_{A} v \mathrm{~d} A\right)^{3}} .
$$

The utilization of momentum coefficient $\beta$ and kinetic energy flux coefficient $\gamma$ is important especially in the case of flue gas injection, where the non-uniformity of velocity profile is significant. The evaluation of $\beta$ and $\gamma$ coefficients is assumed to be based on CFD simulation of incompressible multidimensional flow with mass flow rate based on quasi one-dimensional simulation. Vector of sources responsible for natural draft and minor losses $\mathbf{Q}_{n d}$ is

$$
\mathbf{Q}_{n d}=\left[\begin{array}{c}
A(z) \sigma_{m}(z) \\
p \frac{\mathrm{d} A}{\mathrm{~d} z}-A(z)\left(\rho g+\zeta(z) \rho \frac{v^{2}}{2}\right) \\
0 \\
A(z)\left(\sigma_{q}(z)+\rho g v\right)
\end{array}\right],
$$

where $\sigma_{m}(z)$ is the density of mass source which is connected with mass transfer in the fill zone and $\sigma_{q}(z)$ is the density of heat source connected with heat transfer between water film and moist air in the fill zone. Minor losses in the zones are represented by coefficient $\zeta(z)$, e.g. [8,9] .

Flue gas injection corresponds to a reduction of effective cross-section by the cross-section of flue gas pipe $A_{f g}$. Flue gas mass flow rate is $\dot{m}_{f g}$, the temperature is $t_{f g}$ and specific humidity of flue gas is $x_{f g}$. Let the pressure at flue gas injection is numerically solved, then it is possible to calculate density $\rho_{f g}$ and velocity of flue gas $v_{f g}$ there. Vector of sources responsible for flue gas effect $\mathbf{Q}_{f g}$ is

$$
\mathbf{Q}_{f g}=\frac{\dot{m}_{f g}}{\Delta z_{f g}}\left[\begin{array}{c}
1 \\
v_{f g} \\
w_{a_{f g}} \\
e_{f g}
\end{array}\right],
$$

where $\Delta z_{f g}$ is height of control volume which represents flue gas injection, $w_{a_{f g}}$ is mass fraction of dry air in the flue gas and $e_{f g}$ is total energy of flue gas.

\section{Fill heat and mass transfer}

The model of heat and mass transfer used in this section is modification of model [5]. Integration of the model equations in the fill zone is leading to the evaluation of sources in the governing equations of moist air flow. 


\subsection{Governing equations}

It has been shown in the reference [5] that the change in water mass flow rate with height of the fill $z$ is

$$
\frac{\mathrm{d} \dot{m}_{w}}{\mathrm{~d} z}=\alpha_{m} a_{q} A_{f r}\left(x^{\prime \prime}\left(t_{w}\right)-x_{s}\right),
$$

where $\dot{m}_{w}$ is water mass flow rate, $\alpha_{m}$ is mass transfer coefficient, $a_{q}$ is transfer area per unit volume, $A_{f r}$ is cross sectional area of the fill and $x^{\prime \prime}\left(t_{w}\right)$ is specific moisture of saturated air at water temperature $t_{w}$. The change in water temperature can be expressed as

$$
\frac{\mathrm{d} t_{w}}{\mathrm{~d} z}=\frac{a_{q} A_{f r}}{\dot{m}_{w} c_{p_{w}}}\left[\alpha\left(t_{w}-t\right)+\alpha_{m}\left(x^{\prime \prime}\left(t_{w}\right)-x_{s}\right) l\left(t_{w}\right)\right],
$$

where $\alpha$ is heat transfer coefficient and $l\left(t_{w}\right)$ is latent heat of vaporisation at water temperature. Further modifications of governing equations are based on the definition of Lewis factor [1]

$$
L e_{f}=\frac{\alpha}{\alpha_{m} c_{p}},
$$

where unlike the reference [5] it is important to use dry air mass fraction $w_{a}$, water vapour mass fraction $w_{v}$ and water liquid mass fraction $w_{l}$

$$
c_{p}=w_{a} c_{p_{a}}+w_{v} c_{p_{v}}+w_{l} c_{p_{w}} .
$$

Change in water temperature can be expressed using the definition of Lewis factor as

$$
\frac{\mathrm{d} t_{w}}{\mathrm{~d} z}=\frac{\alpha_{m} a_{q} A_{f r}}{\dot{m}_{w} c_{p_{w}}}\left[L e_{f} c_{p}\left(t_{w}-t\right)+\left(x^{\prime \prime}\left(t_{w}\right)-x_{s}\right) l\left(t_{w}\right)\right],
$$

Merkel number [1] can be in general expressed as

$$
M e=\int_{0}^{A} \frac{\alpha_{m}}{\dot{m}_{w}} \mathrm{~d} A=\int_{0}^{h_{\text {fill }}} \frac{\alpha_{m} a_{q} A_{f r}}{\dot{m}_{w}} \mathrm{~d} z
$$

where $h_{\text {fill }}$ is height of the fill. Averaged value of non dimensional mass transfer coefficient is

$$
\frac{\alpha_{m} a_{q} A_{f r}}{\dot{m}_{w}}=M e / h_{f i l l}
$$

Governing equations for water mass flow rate can be simplified using known value of Merkel number Me [8] as

$$
\begin{gathered}
\frac{\mathrm{d} \dot{m}_{w}}{\mathrm{~d} z}=M e / h_{f i l l} \dot{m}_{w}\left(x^{\prime \prime}\left(t_{w}\right)-x_{s}\right), \\
\frac{\mathrm{d} t_{w}}{\mathrm{~d} z}=\frac{M e / h_{f i l l}}{c_{w}}\left[L e_{f} c_{p}\left(t_{w}-t\right)+\left(x^{\prime \prime}\left(t_{w}\right)-x_{s}\right) l\left(t_{w}\right)\right] .
\end{gathered}
$$

The main difference in comparison to previous model [5] is precise calculation of specific heat capacity, where parameters of supersaturated moist air are taken into account.

\subsection{Calculation of source terms}

Due to different definition of internal energy in this work unlike the work [5], the attention should be paid in expressions for source terms. Because the general mass balance between water and water vapour in infinitesimal control volume of height $\mathrm{d} z$ is

$$
\mathrm{d} \dot{m}_{w}=\dot{m}_{a} \mathrm{~d} x_{s},
$$

we can express mass source as

$$
\sigma_{m}=\frac{\dot{m}_{a}}{A(z)} \frac{\mathrm{d} x}{\mathrm{~d} z}=\frac{\alpha_{m} a_{q} A_{f r}}{A(z)}\left(x^{\prime \prime}\left(t_{w}\right)-x_{s}\right),
$$

and after manipulation

$$
\sigma_{m}=\frac{M e / h_{f i l l}}{A(z)} \dot{m}_{w}\left(x^{\prime \prime}\left(t_{w}\right)-x_{s}\right) .
$$

Heat source is

$$
\sigma_{q}=\frac{\dot{m_{a}}}{A(z)} \frac{\mathrm{d} h_{1+x}}{\mathrm{~d} z}
$$

Finally we get

$$
\sigma_{q}=\frac{\alpha a_{q} A_{f r}}{A(z)}\left(t_{w}-t\right)+h_{v}\left(t_{w}\right) \sigma_{m}
$$

where $h_{v}\left(t_{w}\right)$ is enthalpy of water vapour at water temperature. After application of Lewis factor Le and Merkel number $M e$ we have

$$
\sigma_{q}=L e_{f} \frac{M e / h_{f i l l}}{A(z)} \dot{m}_{w} c_{p}\left(t_{w}-t\right)+h\left(t_{w}\right) \sigma_{m} .
$$

\section{Conclusions}

Proposed model overcomes the drawbacks of previously developed models [4] and [5]. The applicability of the the model to the case of supersaturated moist air is significantly expanded. Finally, the model includes the possibility to simulate flue gas injection, where the approach based on momentum flux coefficient $\beta$, kinetic energy flux coefficient $\gamma$ and additional source vector is used.

\section{Acknowledgement}

This work has been supported by Technology Agency of the Czech Republic under the project Advanced Technologies for Heat and Electricity Production - TE01020036.

\section{References}

1. D. G. Kröger, Air-Cooled Heat Exchangers and Cooling Towers. (Penn Well Corporation, Tulsa, 2004)

2. N. Williamson, M. Behnia, S. Armfield, Int. Journal of Heat and Mass Transfer, 51 (2008), 2227-2236

3. A. K. Majumdar, A. K. Singhal, D. B. Spalding, ASME J. Heat Transfer, 105(4), (1983), 728-735

4. T. Hyhlík, EPJ Web of Conferences, 67, (2014), 02044

5. T. Hyhlík, EPJ Web of Conferences, 92, (2015), 02027

6. S. M. Ghiaasiaan, Two-Phase Flow, Boiling and Condensation in Convectional and Miniature Systems. (Cambridge University Press, 2008)

7. Revised Suplementary Release on Saturation Properties of Ordinary Water Substance. (The International Association for the Properties of Water and Steam, 1992)

8. L. Dvořák, J. Nožička, EPJ Web of Conferences, 67, (2014), 02024

9. J. Stodůlka, R. Vitkovičová, EPJ Web of Conferences, 92, (2015), 02086 